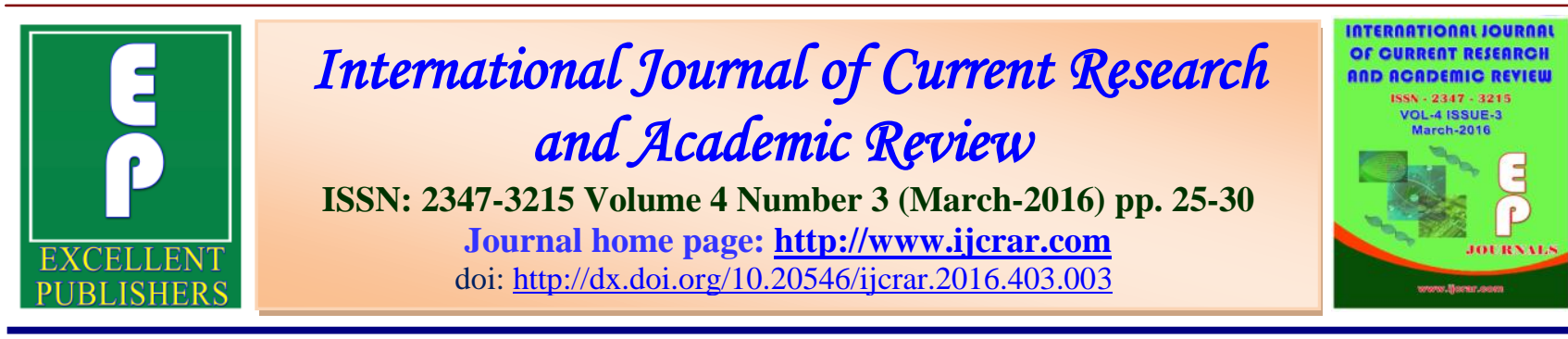

\title{
Correlation of Intrapartum Cardiotocographic Changes with Perinatal Outcome, in cases of Fetal Growth Restriction
}

\author{
Divya Yadav*, Meenal Jain, Saroj Singh, Anu Pathak, Poonam Agarwal \\ 27 MIG, COD Colony, New Shahgang, Agra, India
}

*Corresponding author

\section{KEYWORDS}

Fetal Growth, Intrapartum CTG, Cardiotocographic changes,

Perinatal outcome.

\section{A B S T R A C T}

Fetal Growth Restriction is one of those leading causes of high risk pregnancy, which can result in significant fetal/ perinatal morbidity and mortality if not properly diagnosed and managed. Cardiotocography is a useful and indispesable adjunct to monitor the condition of the endangered foetus. In the proposed study, our focus was to analyse the correlation between the results shown by CTG during intrapartum period and foetal/perinatal outcome in cases of FGR. The present study was conducted in the Department of Obstetrics and Gynecology, S N Medical college, Agra in 2015. Fifty Pateints diagnosed as a case of foetal growth restriction, clinically and by USG with gestation $>32$ weeks who were in active labour, were included in the study. Intrapartum CTG was performed and findings recorded. Where CTG was non reassuring, Meconium stained liquor was present in $80 \%$ cases, cesarean was conducted in $60 \%$, mean birth weight was $1.99 \mathrm{~kg}$, mean Apgar scores both at $1 \mathrm{~min}$ and $5 \mathrm{~min}$ were found to be lower, NICU admissions rate was $20 \%$ and $8 \%$ perinatal deaths all of which occurred with a non ressuring CTG. The perinatal outcome in terms of all above parameters were found to be poor in those cases when CTG was abnormal. On correlating CTG changes with the above parameters, a positive correlation was found which was also statistically significant. Thus Cardiotocography is an effective means for fetal surveillance for pregnancies complicated by fetal growth restriction and an abnormal CTG is an important predictor for poor fetal outcome.

\section{Introduction}

Fetal Growth Restriction is one of those leading causes of high risk pregnancy, which can result in significant fetal/ perinatal morbidity and mortality and finally long term outcome risks, if not properly 
diagnosed and managed. FGR is challenging because of the difficulties in reaching definitive diagnosis of the cause and planning management.

The desire to prevent these complications has prompted the obstetricians to develop methods of assessing fetal condition in utero both ante partum and intrapartum. Cardiotocography is a useful and indispesable adjunct to monitor the condition of the endangered foetus. Cardiotocography, in comparision with intermittent auscultation, results in continuous versus spot like and objective versus subjective data. The introduction of cardiotocography monitoring to the clinical practice has significantly reduced the incidence of birth asphyxia and other fetal complications. It also helps in assessing that whether any timely intervention is required to prevent fetal complications, specially in high risk pregnancies like FGR. Computerised CTG analysis gives an objective assessment, which agrees closely with experienced visual assessment. Also, by being numerical this can be used in the assessment of fetal heart rate analysis in research. There is a correlation between short term variation in fetal heart rate and fetal academia and hyper carbia. In the proposed study, our focus was to analyse the correlation between the results shown by CTG during intrapartum period and foetal/perinatal outcome in cases of FGR.

\section{Materials and Methods}

The present study was conducted in the Deparment of Obstetrics and Gynecology, S N Medical college Agra in 2015.

Fifty Pateints diagnosed as a case of foetal growth restriction, clinically and by USG with gestation >35 weeks who were in active labour, were included in the study.

\section{Exclusion Criteria}

1. Patients with Twin Pregnancy

2. Intrauterine Death

3. Known Congenital Anomaly

4. Wrong Dates and Not Sure of dates

5. Where vaginal delivery is contraindicated

6. Other obsteric complications along with FGR

7. Acute Fetal Distress

8. Abnormal Doppler Findings.

All the patients included in the study underwent detailed history taking, general examination, per abdominal and per vaginal examination to assess patient's condition. A detailed ultrasonography was performed to confirm the gestational age by fetal biometery including BPD and fetal Abdominal circumference. Presentation of the fetus, Amniotic fluid Index was noted and placental localization done. Colour Doppler velocimetery of umbilical, uterine, middle cerebral arteries and Ductus venosus was done. In all intrapartum patients programmed labour protocol was followed and $3^{\text {rd }}$ stage was managed actively.

CTG was performed and findings recorded. Perinatal outcome was noted in the form of Meconium stained liquor, Mode of delivery, Birth weight, Apgar scores at 1 and 5 minutes, need for NICU admissions and finally perinatal deaths.

Cardiotocographic Findings were classified into 3 categories ${ }^{1}$

\section{Reassuring}

Baseline fetal heart between 110-160 beats/ minute

Baseline variability is 5-25 beats/minute. Presence of an acceleration of atleast 15 beats /minute, lasting atleast 15 seconds Absence of deceleration 
Int.J.Curr.Res.Aca.Rev.2016; 4(3): 25-30

Non Reassuring

Tachycardia 160-180 beats /minute

Bradycardia 100-110 beats/ minute

Decreased variability $<5 \mathrm{bpm}$ for $>40 \mathrm{sec}$ but

$>90 \mathrm{sec}$

Early deceleration

Variable Deceleration

Single prolonged deceleration <3minutes

Absence of accelerations

\begin{abstract}
Abnormal
Tachycardia $>180$ beats/ minute

Bradycardia $<100$ beats/ minute

Late deceleration

Atypical Variable deceleration

Prolonged Deceleration $>3$ minutes

Sinusoidal Pattern $>10$ minutes

Absence of accelerations

\section{Results and Discussion}

The results were observed and their correlation of perinatal outcome was studied.
\end{abstract}

\section{Patient Profile}

Mean age:

27.16yrs

Maximum number of cases were multigravida. Mean parity was : 1.66 .

Maximum of cases (68\%) were 36-39 wks of gestation.

Majority of cases (44\%) belonged to class IV of modified B J Prasad classification

\section{Fetal heart rate}

Baseline Fetal Heart Rate pattern

Normal FHR (110-160 bpm):78\%

Abnormal FHR

$: 32 \%$
Tachycardia (>160):24\%

Bradycardia $(<110): 8 \%$

Fetal Heart Rate Variability

Normal Variability (5-25bpm):70\%

Decreased Variability(<5bpm):20\%

Increased Variability(>25bpm):10

While correlating the neonatal outcome in all patients, patients with a reassuring fetal heart rate status, had a mean birth weight of $2.1 \mathrm{Kg}$, had mean Apgar score of 6.26, NICU admissions of $8 \%$, and no perinatal death. In cases, where fetal heart rate status was non reassuring, mean birth weight was slightly lower $1.99 \mathrm{~kg}$, mean Apgar score was 5.56, NICU admissions was $20 \%$, and 4 (8\%) perinatal deaths. All these differences were statistically significant.

The Present study was carried out to study the correlation of intrapartum cardiotocography with perinatal outcome in cases of fetal growth restriction. The study included 50 diagnosed cases of FGR, whose fetal heart rate pattern was ults were monitored by electronic fetal monitor. The results were noted and their correlation with perinatal outcome was studied.

Both the groups were studied for the following parameters of perinatal outcome

Presence of Meconium Stained Liquor

Mode of delivery (vaginal / cesarean)

Birth weight

Apgar score

Admission in neonatal intensive care unit

Perinatal death

The mean age of patients was 27.16 yrs while the mean age in study by Padmagirison et al. ${ }^{4}(2006)$ was 27.7 years. Mean parity was 1.66 in our study. Majority 
of cases belonged to class IV (44\%) socioeconomic class. It is the lower socioeconomic class which is subject to most of risk factor because of poor nutrition and ignorance about various obstetrical problems. In our study $68 \%$ had normal Fetal Heart Rate pattern, (FHR 110-160 bpm) while $32 \%$ had abnormal fetal heart pattern, Tachycardia $(>160)$ in $24 \%$ and Bradycardia $(<110)$ in $8 \%$. In our study, $70 \%$ had normal Variability (5-25bpm), $20 \%$ had decreased Variability $(<5 \mathrm{bpm}), 10 \%$ had Increased Variability $(>25 \mathrm{bpm})$. Of the $28 \%$ paetal heart patients who showed fetal heart rate decelerations, maximum (16\%) had late decelerations. Late decelerations are indicative of uteroplacental insufficiency which is a common complication in FGR fetus. In Mahomed K et al. (1994) study, the late decelerations were the commonest type of pattern in study group. In our study, $14 \%$ had non reassuring fetal heart rate status.

Table 1 depicts that in cases where CTG was reassuring, 34 cases $(85 \%)$ had clear liquor whereas 6 had Meconium stained liquor. Meconium stained liquor as a predictor of perinatal outcome was studied and it was found that MSL is found in distressed foetusus. In our study, where CTG was nonreassuring, $8(80 \%)$ had MSL.

Table.1 Correlation of Intrapartum CTG Changes with MSL

\begin{tabular}{|c|c|c|c|c|c|}
\hline \multirow[t]{2}{*}{ CTG Changes } & \multirow[t]{2}{*}{ Total No } & \multicolumn{2}{|c|}{ Liquor clear } & \multicolumn{2}{|c|}{ MSL } \\
\hline & & No & \%age & No & $\%$ age \\
\hline Reassuring & 40 & 34 & $85 \%$ & 6 & $15 \%$ \\
\hline Non Reassuring & 10 & 2 & $20 \%$ & 8 & $80 \%$ \\
\hline Total & 50 & 36 & & 14 & \\
\hline
\end{tabular}

Table 1 depicts that in cases where CTG was reassuring, 34 cases (85\%) had clear liquor whereas 6 had Meconium stained liquor. Where CTG was non reassuring in 10 subjects, 2 (20\%) had clear liquor and 8 (80\%) had MSL.

Table.2 Correlation of Intrapartum CTG Changes with Mode of Delivery

\begin{tabular}{|l|l|l|l|l|l|}
\hline \multirow{2}{*}{ CTG Changes } & \multirow{2}{*}{ Total No } & \multicolumn{2}{|l|}{ Vaginal Delivery } & \multicolumn{2}{l|}{ Cesarean Delivery } \\
\cline { 3 - 6 } & & No & $\%$ age & No & $\%$ age \\
\hline Reassuring & 40 & 38 & $95 \%$ & 2 & $5 \%$ \\
\hline Non Reassuring & 10 & 4 & $40 \%$ & 6 & $60 \%$ \\
\hline Total & 50 & 42 & $84 \%$ & 8 & $16 \%$ \\
\hline P value $=<0.05$ & & &
\end{tabular}

In cases where CTG was reassuring $(\mathrm{n}=40), 38$ cases $(95 \%)$ had a vaginal delivery and only 2 cases $(5 \%)$ had a cesearean section. When CTG was non reassuring $(\mathrm{n}=10), 4$ cases $(40 \%)$ had a normal vaginal delivery whereas $6(60 \%)$ had a cesarean section. 
Int.J.Curr.Res.Aca.Rev.2016; 4(3): 25-30

Table.3 Perinatal Outcome in Cases of FGR

\begin{tabular}{|l|l|l|}
\hline Neonatal outcome & Reassuring FHR & Non Reassuring FHR \\
\hline Mean Birth Weight & $2.1 \mathrm{Kg}$ & $1.99 \mathrm{Kg}$ \\
\hline Mean Apgar Score & 6.26 in 40 cases & 5.56 in 10 cases \\
\hline NICU Admissions & $4(8 \%)$ & $10(20 \%)$ \\
\hline Perinatal Deaths & 0 & $4(8 \%)$ \\
\hline
\end{tabular}

Table.4 Comparision of Our Study with Other Studies

\begin{tabular}{|l|l|l|l|}
\hline Worker & year & No of high risk cases & Observation \\
\hline Ivanovski et $^{5}{ }^{5}$ & 2005 & 78 & $\begin{array}{l}\text { Reassuring:49 } \\
\text { Non Reassuring:29 }\end{array}$ \\
\hline $\begin{array}{l}\text { Padmagirison } \text { et al } \\
4\end{array}$ & 2006 & 55 & $\begin{array}{l}\text { Reassuring:35 } \\
\text { Non Reassuring:20 }\end{array}$ \\
\hline Piyamongkol ${\text { et } \text { al }^{6}}^{6}$ & 2006 & 270 & $\begin{array}{l}\text { Reassuring:228 } \\
\text { Non Reassuring:42 }\end{array}$ \\
\hline Present study & 2014 & 50 & $\begin{array}{l}\text { Reassuring:40 } \\
\text { Non Reassuring:10 }\end{array}$ \\
\hline
\end{tabular}

Table.5 Comparision with Findings of Other Studies

\begin{tabular}{|l|l|l|}
\hline $\begin{array}{l}\text { Observation s with non reassuring } \\
\text { FHR }\end{array}$ & Other study & Present study \\
\hline 1.MSL & K Mohammad et al:40\% & $80 \%$ \\
\hline 2. Rate of Cesarean & Moga et al:92.9\% & $60 \%$ \\
\hline 3.Lower Apgar Score & Moga et al,Mardinescu & low \\
\hline 4.NICU Admissions & Sood et al:20\% & $20 \%$ \\
\hline 5. Perinatal death & Sood et al: $12 \%$ & $8 \%$ \\
\hline
\end{tabular}

This high incidence of MSL in non reassuring group compared to other studies (40\% in Mohammad et al., 1982) was present probably because we libraly used prostaglandins and oxytocin for labour augmentation. Rate of cesarean was slightly lower in non reassuring group in our study
(60\%) compared to $\mathrm{Moga}^{3}, 2007$ (92.9\%) because we excluded all other obstetric complications. Mean Apgar Score was significantly lower in non reassuring group comparable to M Moga et al. There were $20 \%$ neonates with intrapartum non reassuring fetal heart rate status admitted to 
NICU in our study compared to study by Sood et al. (20\%). There were 4(8\%) perinatal death, all of which occurred in non reassuring group. Major causes of neonatal deaths were birth asphyxia, hypoglycemia, septicemia, respiratory distress syndrome. In a study by A K Sood $(2002)^{2}$, non reactive fetal heart rate patterns were correlated with significant poorer perinatal outcomes in terms of low Apgar scores (32\%), low birth weight (40\%) and perinatal mortality (12\%).

\section{Conclusion}

The perinatal outcome in terms of all above parameters were found to be poor in those cases when CTG was abnormal. On correlating CTG changes with meconium staining, Apgar score, NICU admissions and perinatal deaths, positive correlation was found which was also statistically significant.

Thus Cardiotocography is an effective means for fetal surveillance for pregnancies complicated by fetal growth restriction. An abnormal CTG is an important predictor for poor fetal outcome and is a significant sign to adopt necessary measures to prevent fetal/ perinatal morbidity or mortality. Cardiotocography leads to an increase in obstetric interventions in particular the incidence of cesarean section. This increase in the number of cesarean has been a consequence of poor interpretation of fetal behavior and CTG signals.

\section{References}

American college of obstetrics and gynecologists. October 1999. Antepartum fetal surveillance, Practice Bulletin No 9.

Sood, A.K. 2002. Evaluation of Non stress test in high risk pregnancy. J. Obst. Gynecol. India, 52(2): 71-5.

Moga, M., Piles, L., Martinescu, A. Current interpretation and importance of fetal cardiotocographic monitoring.

Padmagirison, R., Rai, L. 2006. Fetal Doppler versus NST as a predictor of adverse perinatal outcome in severe preeclamsia and fetal growth restriction. $J$. Obstet. Gynecol. India, 56(2): 134-138.

Ivanovski, M.J., Lazarevski, S., Popoviem. 2005. Middle cerebral artery flow velocity waveforms in prediction of adverse outcome in Intrauterine Growth retarted fetusus. Gynecol. Perinatal, 14(3): 133-39.

Piya Mongkol, W., et al. 1999-2002. Comparision of mabual stimulation test and non stress test; A randomized controlled trial. J. Med. Asia Thai, 89(12).

\section{How to cite this article:}

Divya Yadav, Meenal Jain, Saroj Singh, Anu Pathak, Poonam Agarwal. 2016. Correlation of Intrapartum Cardiotocographic Changes with Perinatal Outcome, in Cases of Fetal Growth Restriction. Int.J.Curr.Res.Aca.Rev.4(3): 25-30. doi: http://dx.doi.org/10.20546/ijcrar.2016.403.003 